JOURNAL OF THE AMERICAN MATHEMATICAL SOCIETY

Volume 10, Number 2, April 1997, Pages 371-392

S 0894-0347(97)00229-4

\title{
LATTICE POINTS IN SIMPLE POLYTOPES
}

\author{
MICHEL BRION AND MICHÈLE VERGNE
}

\section{INTRODUCTION}

Consider a convex $n$-dimensional polytope $P$ in $\mathbb{R}^{n}$ with all vertices in the lattice $\mathbb{Z}^{n}$. In this article, we give a formula for the number of lattice points in $P$, in the case where $P$ is simple, that is, if there are exactly $n$ edges through each vertex of $P$. More generally, for any polynomial function $\phi$ on $\mathbb{R}^{n}$, we express

$$
\sum_{m \in \mathbb{Z}^{n} \cap P} \phi(m)
$$

in terms of $\int_{P(h)} \phi(x) d x$ where the polytope $P(h)$ is obtained from $P$ by independent parallel motions of all facets. This extends to simple lattice polytopes the EulerMaclaurin summation formula of Khovanskii and Pukhlikov [8] (valid for lattice polytopes such that the primitive vectors on edges through each vertex of $P$ form a basis of the lattice). As a corollary, we recover results of Pommersheim [9] and Kantor-Khovanskii [6] on the coefficients of the Ehrhart polynomial of $P$. Our proof is elementary. In a subsequent article, we will show how to adapt it to compute the equivariant Todd class of any complete toric variety with quotient singularities.

The Euler-Maclaurin summation formula for simple lattice polytopes has been obtained independently by Ginzburg-Guillemin-Karshon [4]. They used the dictionary between convex polytopes and projective toric varieties with an ample divisor class, in combination with the Riemann-Roch-Kawasaki formula ([1], [7]) for complex manifolds with quotient singularities. A counting formula for lattice points in lattice simplices has been announced by Cappell and Shaneson [2], as a consequence of their computation of the Todd class of toric varieties with quotient singularities.

\section{EULER-MACLAURIN FORMUla FOR POLYTOPES}

Let $V$ be a real vector space of dimension $n$. Let $M$ be a lattice in $V$. Points of $M$ will be called integral points. The vector space $V$ has a canonical Lebesgue measure $d x$ giving volume 1 to a fundamental domain for $M$. More precisely, let $e^{1}, e^{2}, \ldots, e^{n}$ be a basis of $V$ such that

$$
M=\mathbb{Z} e^{1} \oplus \mathbb{Z} e^{2} \oplus \cdots \oplus \mathbb{Z} e^{n} .
$$

If $x=x_{1} e^{1}+x_{2} e^{2}+\cdots+x_{n} e^{n}$ is a point in $V$, then $d x=d x_{1} d x_{2} \cdots d x_{n}$.

Received by the editors October 3, 1996 and, in revised form, October 16, 1996.

1991 Mathematics Subject Classification. Primary 52B20; Secondary 11P21.

Key words and phrases. Simple lattice polytope, Euler-Maclaurin summation formula.

(C) 1997 American Mathematical Society 
We denote by $V^{*}$ the dual vector space to $V$. If $L$ is a lattice in a vector space $W$, we denote by $L^{*}$ its dual lattice in the dual vector space $W^{*}$ :

$$
L^{*}=\left\{y \in W^{*},(x, y) \in \mathbb{Z}, \text { for all } x \in L\right\} .
$$

We will denote $M^{*}$ by $N$. Then $N$ is a lattice in $V^{*}$.

Let $P$ be a convex polytope contained in $V$ with nonempty interior $P^{0}$. We denote by $\operatorname{vol}(P)$ the volume of $P$ with respect to the measure $d x$ on $V$.

We denote by $\mathcal{F}$ the set of closed faces of $P$. We have

$$
\mathcal{F}=\bigcup_{k=0}^{n} \mathcal{F}(k),
$$

where $\mathcal{F}(k)$ is the set of faces of dimension $k$. We have $\mathcal{F}(n)=\{P\}$. By definition, the set $\mathcal{F}(0)$ of extremal points of $P$ is the set of vertices of $P$. The set $\mathcal{F}(1)$ is the set of edges of $P$. A face of codimension 1 is called a facet. A facet $F \in \mathcal{F}(n-1)$ is the intersection of $P$ with an affine hyperplane $\left\{y ;\left(u_{F}, y\right)+\lambda_{F}=0\right\}$. We choose the normal vector $u_{F} \in V^{*}$ to the facet $F$ such that $P$ is contained in $\left\{y,\left(u_{F}, y\right)+\lambda_{F} \geq\right.$ $0\}$. In other words, we choose the inward-pointing normal vector $u_{F}$. This normal vector is determined modulo multiplication by an element of $\mathbb{R}^{+}$.

If $f$ is a face of $P$, we define

$$
\mathcal{F}^{f}=\{F \in \mathcal{F}(n-1) ; f \subset F\} .
$$

We denote by $\langle f\rangle$ the vector space generated by elements $p-q$ where $p \in f$ and $q \in f$. If $f$ is in $\mathcal{F}(k)$, then $\langle f\rangle$ is of dimension $k$.

Definition 2.1. Define $C_{f}$ to be the convex cone generated by elements $p-q$ with $p \in P$ and $q \in f$. We say that $C_{f}$ is the tangent cone to $P$ at its face $f$.

The cone $C_{f}$ is also known as the barrier cone. It contains $\langle f\rangle$ as its largest linear subspace.

Definition 2.2. Define $\sigma_{f}$ to be the polar cone to $C_{f}$ :

$$
\sigma_{f}=\left\{y \in V^{*} \mid(x, y) \geq 0 \text { for all } x \in C_{f}\right\} .
$$

The cone $\sigma_{f}$ is also known as the normal cone.

We have

$$
\sigma_{f}=\sum_{F \in \mathcal{F}^{f}} \mathbb{R}^{+} u_{F}
$$

If $f=\{P\}$, then $\sigma_{f}=\{0\}$.

Definition 2.3. A convex polytope $P$ is said to be simple if there are exactly $n$ edges through each vertex.

For example, in $\mathbb{R}^{3}$, a cube, a pyramid with triangular basis, and a dodecahedron are simple.

Definition 2.4. A convex polytope $P$ is a lattice polytope if all vertices of $P$ are in the lattice $M$.

Consider a convex lattice polytope $P$. We can then choose for each facet $F$ the normal vector $u_{F}$ in the dual lattice $N$. We normalize $u_{F}$ in order that $u_{F}$ be a primitive element of $N$, that is, if $t u_{F} \in N$, then $t$ is an integer. 
Let us number facets of $P$ as $F_{1}, F_{2}, \ldots, F_{d}$. We denote by $u_{i} \in N$ the normalized normal vector $u_{F_{i}}$ to $F_{i}$. Let $\lambda_{i}=\lambda_{F_{i}}$. Thus $P$ is the intersection of $d$ half-spaces:

$$
P=\left\{x \in V,\left(u_{i}, x\right)+\lambda_{i} \geq 0,1 \leq i \leq d\right\} .
$$

Consider $h \in \mathbb{R}^{d}, h=\left(h_{1}, h_{2}, \ldots, h_{d}\right)$. For $h \in \mathbb{R}^{d}$, define

$$
P(h)=\left\{x \in V,\left(u_{i}, x\right)+\lambda_{i}+h_{i} \geq 0,1 \leq i \leq d\right\} .
$$

Then $P(h)$ is a convex polytope. Moreover, for small $h, P(h)$ and $P$ have the same directions of faces. In particular, $P(h)$ is simple if $P$ is simple and $h$ is small enough.

Let $C$ be a closed convex cone in a vector space $W$ with a lattice $L$. We denote by $\langle C\rangle$ the vector space spanned by $C$. The dimension of $C$ is defined to be equal to the dimension of the vector space $\langle C\rangle$. We say that $C$ is acute (or pointed) if $C$ does not contain any nonzero linear subspace. A cone $C$ is said to be polyhedral (respectively, rational polyhedral) if $C$ is generated by a finite number of elements of $W$ (respectively, of $L$ ). An acute polyhedral cone $C$ of dimension $k$ is said to be simplicial if $C$ has exactly $k$ edges. If $P$ is a convex lattice polytope, then cones $C_{f}$ associated to faces $f$ of $P$, and their polar cones $\sigma_{f}$ are rational and polyhedral. The cone $C_{f}$ is acute if and only if $f$ is a vertex of $P$. The cones $\sigma_{f}$ are acute for all $f \in \mathcal{F}$.

A finite collection $\Sigma$ of rational polyhedral acute cones in $V^{*}$ is called a fan if

1) for any face $\tau$ of an element $\sigma \in \Sigma$, we have $\tau \in \Sigma$;

2) for $\sigma, \tau \in \Sigma$, we have $\sigma \cap \tau \in \Sigma$.

The fan is complete if $\bigcup_{\sigma \in \Sigma} \sigma=V^{*}$.

We denote by $\Sigma(k)$ the set of cones in the fan $\Sigma$ of dimension $k$.

If $P$ is a convex lattice polytope, the collection $\Sigma_{P}=\left\{\sigma_{f}, f \in \mathcal{F}\right\}$ is a complete fan, called the normal fan of $P$ ([11]). If $f \in \mathcal{F}(n-k)$ is a face of codimension $k$ of $P$, then $\sigma_{f}$ has dimension $k$. The fan $\Sigma_{P}$ depends only on the directions of the faces of $P$. In particular, the homothetic polytope $q P$ ( $q$ a positive integer) has the same fan as $P$.

Definition 2.5. A fan $\Sigma$ is said to be simplicial if each cone $\sigma \in \Sigma$ is simplicial.

A lattice polytope is simple if and only if its fan is simplicial.

Let $\Sigma$ be a simplicial fan. Let $d$ be the cardinal of $\Sigma(1)$. We denote elements of $\Sigma(1)$ as $\ell_{1}, \ell_{2}, \ldots, \ell_{d}$. Let $u_{i}, 1 \leq i \leq d$, be the primitive integral vector (with respect to $N$ ) on the half-line $\ell_{i}$.

Definition 2.6. Let $\sigma \in \Sigma$. We denote by $\mathcal{E}(\sigma)$ the subset of the set $\{1,2, \ldots, d\}$ consisting of those $i$ such that the half-line $\ell_{i}$ is an edge of $\sigma$.

The elements $\left\{u_{i}, i \in \mathcal{E}(\sigma)\right\}$ are linearly independent. Let

$$
U(\sigma)=\bigoplus_{i \in \mathcal{E}(\sigma)} \mathbb{Z} u_{i}
$$

and

$$
T(\sigma)=\langle\sigma\rangle / U(\sigma) .
$$

Let $k$ be the dimension of $\sigma$. Then $U(\sigma)=\mathbb{Z}^{k}$ is a lattice in $\langle\sigma\rangle=\mathbb{R}^{k}$, and $T(\sigma)=\mathbb{R}^{k} / \mathbb{Z}^{k}$ is a $k$-dimensional torus. 
Consider the lattice $N(\sigma)=N \cap\langle\sigma\rangle$ of $\langle\sigma\rangle$. Then $U(\sigma)$ is a sublattice of $N(\sigma)$, which is usually different from $N(\sigma)$. Define

$$
G(\sigma)=N(\sigma) / U(\sigma) .
$$

Then $G(\sigma)$ is a finite subgroup of $T(\sigma)$. The order of $G(\sigma)$ is called the multiplicity of $\sigma$ in toric geometry.

If $\tau$ is a face of $\sigma$, we have $U(\tau)=\langle\tau\rangle \cap U(\sigma)$ since $\mathcal{E}(\tau) \subset \mathcal{E}(\sigma)$ and the elements $u_{i}, i \in \mathcal{E}(\sigma)$, are linearly independent. Thus we have a natural inclusion $T(\tau) \subset T(\sigma)$. This induces a natural inclusion of the finite group $G(\tau)$ in $G(\sigma)$. By the definition of $u_{i}$ as a primitive vector, the group $G(\sigma)$ is trivial if $\sigma \in \Sigma(1)$.

Definition 2.7. Let $\Sigma$ be a simplicial fan. We denote by $T_{\Sigma}$ the set obtained from the disjoint union of the tori $T(\sigma)(\sigma \in \Sigma)$ by identifying the subsets $T(\sigma \cap \tau)$ of $T(\sigma)$ and $T(\tau)$ for all $(\sigma, \tau) \in \Sigma \times \Sigma$.

We write $T_{\Sigma}=\bigcup_{\sigma \in \Sigma} T(\sigma)$. In $T_{\Sigma}$, we have $T(\sigma) \cap T(\tau)=T(\sigma \cap \tau)$. A visual way to represent the set $T_{\Sigma}$ associated to a rational fan $\Sigma$ is the following. We denote by $Q(\sigma)$ the subset

$$
Q(\sigma)=\sum_{i \in \mathcal{E}(\sigma)}\left[0,1\left[u_{i}\right.\right.
$$

of $\langle\sigma\rangle$. It is clear that the map $Q(\sigma) \mapsto T(\sigma)$ (restriction of the quotient map $\langle\sigma\rangle \rightarrow\langle\sigma\rangle / U(\sigma))$ is an isomorphism. Furthermore, $Q(\sigma) \cap Q(\tau)=Q(\sigma \cap \tau)$. Consider the subset $Q_{\Sigma}$ of $V^{*}$ defined by

$$
Q_{\Sigma}=\bigcup_{\sigma \in \Sigma} Q(\sigma)
$$

Then the set $Q_{\Sigma}$ is isomorphic to the set $T_{\Sigma}$.

Consider the finite subgroup $G(\sigma) \subset T(\sigma)$.

Definition 2.8. The subset $\Gamma_{\Sigma}$ of $T_{\Sigma}$ is defined to be

$$
\Gamma_{\Sigma}=\bigcup_{\sigma \in \Sigma} G(\sigma)
$$

Thus we can think of $\Gamma_{\Sigma}$ as the union of all finite groups $G(\sigma)(\sigma \in \Sigma)$ with equivalence relations given by $G(\sigma \cap \tau)=G(\sigma) \cap G(\tau) \subset T_{\Sigma}$. In particular, the neutral elements of all the groups $G(\sigma)$ are identified to a unique element of $\Gamma_{\Sigma}$, denoted by 1 . In the identification of $T_{\Sigma}$ with the subset $Q_{\Sigma}$ of $V^{*}$, the subset $\Gamma_{\Sigma}$ of $T_{\Sigma}$ is identified with $Q_{\Sigma} \cap N$.

Example 2.9. Let $a, b, c$ be pairwise coprime integers, and let $P(a, b, c)$ be the simplex in $\mathbb{R}^{3}$ with vertices

$$
O=(0,0,0), \quad A=(a, 0,0), \quad B=(0, b, 0), \quad C=(0,0, c) .
$$

The rational fan $\Sigma_{P}$ associated to $P$ has edges

$$
\ell_{1}=\mathbb{R}^{+} e_{1}, \quad \ell_{2}=\mathbb{R}^{+} e_{2}, \quad \ell_{3}=\mathbb{R}^{+} e_{3}, \quad \ell_{0}=\mathbb{R}^{+}\left(-b c e_{1}-c a e_{2}-a b e_{3}\right),
$$

where $\left(e_{1}, e_{2}, e_{3}\right)$ is the canonical basis of $\left(\mathbb{R}^{3}\right)^{*}$. Let us list the nontrivial abelian groups $G(\sigma)$ for $\sigma \in \Sigma_{P}$. Denote by $G(j, \ldots, k)$ the group associated to a cone in $\Sigma_{P}$ generated by $\left(\ell_{j}, \ldots, \ell_{k}\right)$. We have

$$
G(0,2,3)=\mathbb{Z} / b c \mathbb{Z}, \quad G(0,3,1)=\mathbb{Z} / c a \mathbb{Z}, \quad G(0,1,2)=\mathbb{Z} / a b \mathbb{Z}
$$


and

$$
G(0,1)=\mathbb{Z} / a \mathbb{Z}, \quad G(0,2)=\mathbb{Z} / b \mathbb{Z}, \quad G(0,3)=\mathbb{Z} / c \mathbb{Z} .
$$

Our set $\Gamma_{\Sigma}$ is equal to

$$
\Gamma=(\mathbb{Z} / b c \mathbb{Z}) \cup(\mathbb{Z} / a c \mathbb{Z}) \cup(\mathbb{Z} / a b \mathbb{Z}),
$$

where we identify the common subsets $\mathbb{Z} / a \mathbb{Z}, \mathbb{Z} / b \mathbb{Z}, \mathbb{Z} / c \mathbb{Z}$.

Definition 2.10. A simple lattice polytope $P$ is called a Delzant polytope if each cone $\sigma \in \Sigma_{P}$ is spanned by a part of a basis of $N$, i.e., if $G(\sigma)=\{1\}$ for each element $\sigma \in \Sigma_{P}$.

Equivalently, $P$ is a Delzant polytope if the set $\Gamma_{\Sigma}$ constructed from the complete fan $\Sigma_{P}$ of $P$ is reduced to $\{1\}$. This is a very strong hypothesis. As shown by the example above, many lattice simplices are not Delzant. As another example, consider the lattice simplex $P(a)$ in $\mathbb{R}^{3}$ with vertices $(0,0,0),(1,0,0),(0,1,0)$, $(1,1, a)$, where $a \geq 2$ is an integer. Then $P(a)$ is not Delzant, and the only lattice points in $P(a)$ are its vertices. It follows that $P(a)$ is not a union of Delzant polytopes.

Remark 2.11. In the dictionary (that we do not use here) between convex polytopes and toric varieties, a projective toric variety with quotient singularities is associated to a simple lattice polytope $P$. This toric variety is nonsingular if and only if the polytope $P$ is a Delzant polytope (see [3]).

We now define for $k \in\{1,2, \ldots, d\}$ functions $a^{k}$ on $T_{\Sigma}$ associated to the $d$ elements $\ell_{k}$ of $\Sigma(1)$. The torus $T(\sigma)=\langle\sigma\rangle / U(\sigma)$ comes equipped with a basis of its lattice of characters: for each $k \in \mathcal{E}(\sigma)$, we define $\chi_{\sigma}^{k}(g)=e^{2 i \pi y_{\sigma}^{k}}$ if $y=$ $\sum_{j \in \mathcal{E}(\sigma)} y_{\sigma}^{j} u_{j}$ is an element of $\langle\sigma\rangle$ representing $g$.

The following lemma is obvious.

Lemma 2.12. For any $k \in\{1,2, \ldots, d\}$, there exists a unique function $a^{k}: T_{\Sigma} \rightarrow$ $\mathbb{C}^{*}$ such that

1) if $k \notin \mathcal{E}(\sigma)$, then $a^{k}(g)=1$ for all $g \in T(\sigma) \subset T_{\Sigma}$;

2) if $k \in \mathcal{E}(\sigma)$, then $a^{k}(g)=\chi_{\sigma}^{k}(g)$ if $g \in T(\sigma) \subset T_{\Sigma}$.

Observe that there exists a unique continuous function $\xi^{k}$ on $V^{*}$ which is linear on each cone of $\Sigma$, and such that $\xi^{k}\left(u_{k}\right)=1$ and $\xi^{k}\left(u_{j}\right)=0$ for all $j \neq k$. Let us identify $T_{\Sigma}$ with the subset $Q_{\Sigma}$ of $V^{*}$. Then if $g \in T_{\Sigma}$ is represented by the element $y \in Q_{\Sigma}$, we have $a^{k}(g)=e^{2 i \pi \xi^{k}(y)}$.

We can characterize the subset $G(\sigma)$ of $\Gamma_{\Sigma}$ as follows.

Lemma 2.13. Let $\sigma \in \Sigma$. We have

$$
G(\sigma)=\left\{\gamma \in \Gamma_{\Sigma}, a^{k}(\gamma)=1 \text { for all } k \notin \mathcal{E}(\sigma)\right\}
$$

Now we turn to the definition of Todd operators. Consider the analytic function

$$
\operatorname{Todd}(z)=\frac{z}{1-\exp (-z)}=1+\frac{1}{2} z+\sum_{k=1}^{\infty}(-1)^{k-1} \frac{B_{k}}{(2 k) !} z^{2 k}
$$

where $B_{k}$ are the Bernoulli numbers.

Let $a$ be a complex number. Consider more generally the function

$$
\operatorname{Todd}(a, z)=\frac{z}{1-a \exp (-z)} \text {. }
$$


This function is analytic in a neighborhood of 0 . Consider its Taylor expansion

$$
\operatorname{Todd}(a, z)=\sum_{k=0}^{\infty} c(a, k) z^{k}
$$

for $z$ small.

Let $h$ be a real variable. For any $a \in \mathbb{C}$, consider the operator

$$
\operatorname{Todd}(a, \partial / \partial h)=\sum_{k=0}^{\infty} c(a, k)(\partial / \partial h)^{k} .
$$

We have

$$
\operatorname{Todd}(1, \partial / \partial h)=1+\frac{1}{2} \partial / \partial h+\sum_{k=1}^{\infty}(-1)^{k-1} \frac{B_{k}}{(2 k) !}(\partial / \partial h)^{2 k},
$$

while for $a \neq 1$,

$$
\operatorname{Todd}(a, \partial / \partial h)=(1-a)^{-1} \partial / \partial h+\sum_{k=2}^{\infty} c(a, k)(\partial / \partial h)^{k} .
$$

We denote $\operatorname{Todd}(1, \partial / \partial h)$ simply by $\operatorname{Todd}(\partial / \partial h)$. If $\phi$ is a polynomial function of $h$, then $\operatorname{Todd}(a, \partial / \partial h) \phi(h)$ is well defined, as $(\partial / \partial h)^{k} \phi=0$ for large $k$.

Definition 2.14. Let $\Sigma$ be a complete simplicial fan. For $g \in T_{\Sigma}$, define

$$
\operatorname{Todd}(g, \partial / \partial h)=\prod_{k=1}^{d} \operatorname{Todd}\left(a^{k}(g), \partial / \partial h_{k}\right) .
$$

Define

$$
\operatorname{Todd}(\Sigma, \partial / \partial h)=\sum_{\gamma \in \Gamma_{\Sigma}} \operatorname{Todd}(\gamma, \partial / \partial h) .
$$

Recall a version of the Euler-Maclaurin formula. If $\phi(x)$ is a polynomial function on $\mathbb{R}$ and $s \leq t$ are integers, then

$$
\sum_{k=s}^{t} \phi(k)=\left.\operatorname{Todd}\left(\partial / \partial h_{1}\right) \operatorname{Todd}\left(\partial / \partial h_{2}\right)\left(\int_{s-h_{1}}^{t+h_{2}} \phi(x) d x\right)\right|_{h_{1}=h_{2}=0} .
$$

We will generalize this formula to simple lattice polytopes.

Let $P$ be a simple lattice polytope with $d$ facets. Let $|M \cap P|$ be the number of lattice points in $P$, and $\left|M \cap P^{0}\right|$ the number of lattice points in the interior $P^{0}$ of $P$. Let $P(h)$ be the deformed polytope obtained from $P$ after $d$ independent parallel motions of its facets (formula (2.2)). The main theorem of this article is

Theorem 2.15. Let $V$ be a vector space with a lattice $M$. Let $P$ be a simple lattice polytope in $V$, and let $\Sigma$ be its associated fan. Then, for small $h$, the volume $\operatorname{vol}(P(h))$ of the deformed polytope $P(h)$ is a polynomial function of $h$, and we have

$$
|M \cap P|=\left.\operatorname{Todd}(\Sigma, \partial / \partial h) \operatorname{vol}(P(h))\right|_{h=0},
$$

while

$$
\left|M \cap P^{0}\right|=\left.\operatorname{Todd}(\Sigma,-\partial / \partial h) \operatorname{vol}(P(h))\right|_{h=0} .
$$

More generally, if $\phi$ is a polynomial function on $V$, then

$$
I(\phi)(h)=\int_{P(h)} \phi(x) d x
$$


is a polynomial function of $h$ for small $h$, and

$$
\sum_{m \in M \cap P} \phi(m)=\left.\operatorname{Todd}(\Sigma, \partial / \partial h) I(\phi)(h)\right|_{h=0},
$$

while

$$
\sum_{m \in M \cap P^{0}} \phi(m)=\left.\operatorname{Todd}(\Sigma,-\partial / \partial h) I(\phi)(h)\right|_{h=0} .
$$

Remark 2.16. If, moreover, $P$ is a Delzant polytope, then the corresponding set $\Gamma_{\Sigma}$ is reduced to $\{1\}, \operatorname{Todd}(\Sigma, \partial / \partial h)$ is the usual Todd operator considered by Khovanskii and Pukhlikov [8] and Theorem 2.15 is due to them in this case.

We will prove Theorem 2.15 in the next section.

\section{INTEGRAL FORMULAS}

As an example of our method, let us first prove identity (2.8). It will be convenient to extend the action of Todd operators to exponential functions $h \mapsto e^{h z}$, for $z$ a small complex number. Indeed, for $z$ small, the series

$$
\operatorname{Todd}(\partial / \partial h) e^{h z}=e^{h z}\left(1+\frac{1}{2} z+\sum_{k=1}^{\infty}(-1)^{k-1} \frac{B_{k}}{(2 k) !} z^{2 k}\right)
$$

is convergent and equal to $\operatorname{Todd}(z) e^{h z}$.

Let $[s, t]$ be an interval. Then we have

$$
\int_{s}^{t} e^{z x} d x=\frac{e^{t z}}{z}-\frac{e^{s z}}{z}
$$

Assume $t$ and $s$ are integers; then

$$
\sum_{k=s}^{t} e^{k z}=e^{s z}\left(1+e^{z}+\cdots+e^{(t-s) z}\right)=e^{s z} \frac{1-e^{(t-s+1) z}}{1-e^{z}}
$$

that is,

$$
\sum_{k=s}^{t} e^{k z}=\frac{e^{t z}}{1-e^{-z}}+\frac{e^{s z}}{1-e^{z}}
$$

On the other hand,

$$
\int_{s-h_{1}}^{t+h_{2}} e^{z x} d x=e^{h_{2} z} \frac{e^{t z}}{z}-e^{-h_{1} z} \frac{e^{s z}}{z}
$$

Therefore

$$
\begin{gathered}
\left.\operatorname{Todd}\left(\partial / \partial h_{1}\right) \operatorname{Todd}\left(\partial / \partial h_{2}\right)\left(\int_{s-h_{1}}^{t+h_{2}} e^{z x} d x\right)\right|_{h_{1}=h_{2}=0} \\
=\operatorname{Todd}(z) \frac{e^{t z}}{z}-\operatorname{Todd}(-z) \frac{e^{s z}}{z}
\end{gathered}
$$

Comparing with formula (3.2), we obtain

$$
\left.\operatorname{Todd}\left(\partial / \partial h_{1}\right) \operatorname{Todd}\left(\partial / \partial h_{2}\right)\left(\int_{s-h_{1}}^{t+h_{2}} e^{z x} d x\right)\right|_{h_{1}=h_{2}=0}=\sum_{k=s}^{t} e^{k z}
$$


If we take the Taylor expansion at the origin of this identity in $z$, we obtain formula (2.8).

Our proof of Theorem 2.15 for an $n$-dimensional lattice polytope $P$ will be based on the same approach. Let $y \in V_{\mathbb{C}}^{*}$, and let $P \subset V$ be a polytope (not necessarily a lattice polytope). Define

$$
E(P)(y)=\int_{P} e^{(x, y)} d x .
$$

Then the volume of $P$ is the value of $E(P)$ at $y=0$.

If, moreover, $P$ is a lattice polytope, define

$$
D(P)(y)=\sum_{m \in M \cap P} e^{(m, y)}
$$

and

$$
D\left(P^{0}\right)(y)=\sum_{m \in M \cap P^{0}} e^{(m, y)} .
$$

Then the number $|M \cap P|$ of lattice points in $P$ is the value of $D(P)$ at $y=$ 0 . Although $E(P)(y), D(P)(y)$ and $D\left(P^{0}\right)(y)$ are analytic functions of $y$, "simple" expressions (similar to formulae (3.1) and (3.2)) for $E(P)(y), D(P)(y)$ and $D\left(P^{0}\right)(y)$ will be given only when $P$ is simple and $y$ is "generic". On this formula for $E(P(h))(y)$, it will be easy to analyze the action of the Todd operator $\operatorname{Todd}(\Sigma, \partial / \partial h)$ and to compare it with $D(P)(y)$.

Recall that $C_{f}$ denotes the tangent cone to $P$ at its face $f$. Choose $v_{0} \in f$. Set

$$
C_{P}^{+}(f)=v_{0}+C_{f} .
$$

As $C_{f}$ is invariant by translation by vectors in $\langle f\rangle$, the affine cone $C_{P}^{+}(f)$ does not depend of the choice of $v_{0} \in f$. We call it the inward pointing affine cone tangent to $P$ at $f$. Thus $C_{P}^{+}(f)$ contains $P$ and $P=\bigcap_{f \in \mathcal{F}} C_{P}^{+}(f)$.

Let

$$
C_{P}^{-}(f)=v_{0}-C_{f}
$$

be the outward pointing affine cone at $f$.

If $E$ is a subset of $V$, we denote by $\chi_{E}$ its characteristic function.

Proposition 3.1. Let $P$ be a convex polytope with non empty interior $P^{0}$. Then we have the identities

(1)

$$
\chi_{P}=\sum_{f \in \mathcal{F}}(-1)^{\operatorname{dim} f} \chi_{C_{P}^{+}(f)},
$$

$$
(-1)^{n} \chi_{P^{0}}=\sum_{f \in \mathcal{F}}(-1)^{\operatorname{dim} f} \chi_{C_{P}^{-}(f)},
$$

$$
\chi_{\{0\}}=\sum_{f \in \mathcal{F}}(-1)^{\operatorname{dim} f} \chi_{C_{f}} .
$$


Proof. A version of these identities can be found in [5]. We give another proof, based on the Euler identities

$$
\sum_{f \in \mathcal{F}}(-1)^{\operatorname{dim} f}=1
$$

and, for any point $m$ in the boundary of $P$,

$$
\sum_{f \in \mathcal{F}, m \in f}(-1)^{\operatorname{dim} f}=0 .
$$

Let $m$ be an arbitrary point of $V$. We have to prove the relations

(1) If $m \in P$, then

$$
\sum_{f \in \mathcal{F}, m \in C_{P}^{+}(f)}(-1)^{\operatorname{dim} f}=1
$$

(2) If $m \notin P$, then

$$
\sum_{f \in \mathcal{F}, m \in C_{P}^{+}(f)}(-1)^{\operatorname{dim} f}=0
$$

(3) If $m \in P^{0}$, then

$$
\sum_{f \in \mathcal{F}, m \in C_{P}^{-}(f)}(-1)^{\operatorname{dim} f}=(-1)^{n} .
$$

(4) If $m \notin P^{0}$, then

$$
\sum_{f \in \mathcal{F}, m \in C_{P}^{-}(f)}(-1)^{\operatorname{dim} f}=0 .
$$

$$
\sum_{f \in \mathcal{F}, 0 \in C_{f}}(-1)^{\operatorname{dim} f}=1
$$

(6) If $m \neq 0$, then

$$
\sum_{f \in \mathcal{F}, m \in C_{f}}(-1)^{\operatorname{dim} f}=0 .
$$

First observe that $m \in P$ if and only if $m \in C_{P}^{+}(f)$ for all $f \in \mathcal{F}$. So assertion (1) is just the Euler identity (3.4), and the same holds for (5). For (3), if $m \in P^{0}$, then the unique face $f$ such that $m \in C_{P}^{-}(f)$ is $f=P$.

Let us prove (4). Let $m \notin P^{0}$. Consider the convex hull $H$ of $P$ and $m$. Let $\mathcal{F}(H)$ be the set of faces of $H$. Let $\mathcal{F}_{m}(H)$ be the set of faces of $H$ containing $m$. Write $\mathcal{F}(H)$ as the disjoint union $\mathcal{F}_{m}(H) \cup \mathcal{F}_{n}(H)$. Using relations (3.4), (3.5) for the polytope $H$ and its boundary point $m$, we obtain $\sum_{g \in \mathcal{F}_{n}(H)}(-1)^{\operatorname{dim} g}=1$. It is easy to see that the faces $g$ in $\mathcal{F}_{n}(H)$ are faces of $P$ and that these are all the faces $f$ of $P$ such that $m \notin C_{P}^{-}(f)$. Thus we have $\sum_{f \in \mathcal{F}, m \notin C_{P}^{-}(f)}(-1)^{\operatorname{dim} f}=1$. Subtracting the Euler identity for $P$, we obtain (4).

Let us prove (2). Let $m \notin P$. Consider the convex hull $H$ of $P$ and $m$. Let $R$ be the closure of $H \backslash P$. The set $R$ is not convex in general; however, it can be contracted to $m$. Therefore, the Euler identities holds for $R$. Let $\mathcal{F}(R)$ be the set 
of faces of $R$. Let $\mathcal{F}_{m}(R)$ be the set of faces of $R$ containing $m$. Write $\mathcal{F}(R)$ as the disjoint union $\mathcal{F}_{m}(R) \cup \mathcal{F}_{n}(R)$. Using relations (3.4), (3.5) for the polytope $R$ and its vertex $m$, we obtain $\sum_{g \in \mathcal{F}_{n}(R)}(-1)^{\operatorname{dim} g}=1$. It is easy to see that the faces $g$ in $\mathcal{F}_{n}(R)$ are faces of $P$ and that these are all the faces $f$ of $P$ such that $m \notin C_{P}^{+}(f)$. Thus we have $\sum_{f \in \mathcal{F}, m \notin C_{P}^{+}(f)}(-1)^{\operatorname{dim} f}=1$. Subtracting the Euler identity for $P$, we obtain relation (2).

Finally, let us prove (6). We may assume that 0 is an interior point of $P$. Let $m \neq 0$. Choose a small positive number $t$. Recall that $C_{t P}^{+}(t f)$ denotes the inward pointing affine cone for the face $t f$ of $t P$, where $t$ is a small positive number. Then there exists $t$ sufficiently small such that $m \in C_{f}$ if and only if $m \in C_{t P}^{+}(t f)$. Thus the last relation is deduced from relation (2) by considering the polytope $t P$ for $t$ sufficiently small.

To a point $m$ of $V$, we associate its $\delta$-measure $\delta(m)$, defined as follows. For any continuous function $\phi$ on $V$, we have $(\delta(m), \phi)=\phi(m)$. If $S$ is a discrete subset of $V$, we denote by $\delta(S)=\sum_{s \in S} \delta(s)$ its $\delta$-measure.

The following proposition follows immediately from Proposition 3.1.

Proposition 3.2. Let $P$ be a convex lattice polytope. We have the equalities

$$
\begin{gathered}
\delta(M \cap P)=\sum_{f \in \mathcal{F}}(-1)^{\operatorname{dim} f} \delta\left(M \cap C_{P}^{+}(f)\right), \\
(-1)^{n} \delta\left(M \cap P^{0}\right)=\sum_{f \in \mathcal{F}}(-1)^{\operatorname{dim} f} \delta\left(M \cap C_{P}^{-}(f)\right),
\end{gathered}
$$

$$
\delta(\{0\})=\sum_{f \in \mathcal{F}}(-1)^{\operatorname{dim} f} \delta\left(M \cap C_{f}\right) .
$$

We will consider Fourier transforms of the measures $\delta\left(M \cap C_{f}\right)$. They make sense in the framework of generalized functions. We will use the function notation $\Theta(y)$ for a generalized function $\Theta$ on $V^{*}$, although the value of $\Theta$ at a particular point $y$ may not have a meaning. We denote by $\int_{V^{*}} \Theta(y) \phi(y) d y$ the value of $\Theta$ on a test density $\phi(y) d y$. We will say that $\Theta$ is smooth on an open subset $U$ of $V^{*}$ if there exists a smooth function $\theta(y)$ on $U$ such that $\int_{V^{*}} \Theta(y) \phi(y) d y=\int_{V^{*}} \theta(y) \phi(y) d y$ for all test functions $\phi$ with compact support contained in $U$. Then the value of $\Theta$ at $y \in U$ is defined to be $\theta(y)$. If there exist two smooth functions $f, g$ on $V^{*}$, with $g$ not identically 0 , such that the equation $g(y) \Theta(y)=f(y)$ holds in the space of generalized functions on $V^{*}$, then $\Theta$ is smooth on the open set $U=\{y, g(y) \neq 0\}$ and $\Theta(y)=f(y) / g(y)$ on $U$.

Consider for example $V=\mathbb{R}$. Consider the discrete measure $\delta(\mathbb{Z})=\sum_{n \in \mathbb{Z}} \delta(n)$. We denote its Fourier transform by $\Theta(y)=\sum_{k \in \mathbb{Z}} e^{i k y}$. This means that the generalized function $\Theta(y)$ is the limit in the space of generalized functions of the smooth functions $\sum_{|k| \leq K} e^{i k y}$. We have thus for a smooth test function $\phi$ on $\mathbb{R}$

$$
\int_{\mathbb{R}} \Theta(y) \phi(y) d y=\sum_{k \in \mathbb{Z}} \int_{\mathbb{R}} e^{i k y} \phi(y) d y .
$$


Clearly $\left(1-e^{i y}\right)\left(\sum_{k \in \mathbb{Z}} e^{i k y}\right)=0$ so that $\Theta(y)$ is supported on $2 \pi \mathbb{Z}$. In fact, Poisson summation formula is

$$
(2 \pi)^{-1} \int_{\mathbb{R}} \Theta(y) \phi(y) d y=\sum_{k \in \mathbb{Z}} \phi(2 \pi k) .
$$

Let

$$
\mathbb{Z}^{+}=\{0,1,2,3, \ldots\} .
$$

Let $a$ be a complex number of modulus 1 . Consider the discrete measure $h(a)=$ $\sum_{n \in \mathbb{Z}^{+}} a^{n} \delta(n)$ and its Fourier transform $\Theta_{a}^{+}(y)=\sum_{n \in \mathbb{Z}^{+}} a^{n} e^{i n y}$. We have the equality

$$
\left(1-a e^{i y}\right) \Theta_{a}^{+}(y)=1 .
$$

Thus the generalized function $\Theta_{a}^{+}(y)$ is smooth outside the set $i \log a+2 \pi \mathbb{Z}$, and for $y \notin i \log a+2 \pi \mathbb{Z}$,

$$
\Theta_{a}^{+}(y)=\frac{1}{1-a e^{i y}}
$$

In particular, $\Theta_{a}^{+}(y)$ is a rational function of $e^{i y}$. We will generalize this formula to higher dimensions.

We call a meromorphic function $\Phi(y)$ on $V_{\mathbb{C}}^{*}$ a rational function of $e^{y}$ if for some basis $\left(e_{1}, \ldots, e_{n}\right)$ of $N$, writing $y=\sum_{i=1}^{n} y_{i}^{*} e_{i}, \Phi\left(y_{1}^{*}, \ldots, y_{n}^{*}\right)$ is a rational function of $e^{y_{1}^{*}}, \ldots, e^{y_{n}^{*}}$. This does not depend on the choice of the basis of $N$.

Definition 3.3. Let $C$ be a rational polyhedral convex cone in $V$. Denote by $\Theta(C)(y)$ the Fourier transform of $\delta(M \cap C)$ :

$$
\Theta(C)(y)=\sum_{m \in M \cap C} e^{i(m, y)} .
$$

Proposition 3.4. Let $C$ be a rational polyhedral convex cone in $V$; let $W$ be the largest linear subspace contained in $C$.

(1) The generalized function $\Theta(C)$ is supported on a discrete union of translates of $W^{\perp}$.

(2) If $C$ is acute (i.e. $W=0$ ), then there exists a meromorphic function $\phi$ on $V_{\mathbb{C}}^{*}$ such that for $y$ outside a union of a discrete set of affine hyperplanes

$$
\Theta(C)(y)=\phi(i y) .
$$

The order at 0 of the function $\phi$ is at least $-n$. Moreover, $\phi(y)$ is a rational function of $e^{y}$.

Proof. Observe that $W$ is a rational subspace of $V$. Moreover, for any $m_{0} \in M \cap W$, $\left(1-e^{i\left(m_{0}, y\right)}\right) \Theta(C)(y)=0$ as $M \cap C$ is invariant under translation by elements of $M \cap W$. Hence the support of $\Theta$ is contained in the set

$$
\left\{y \in V^{*} \mid\left(y, m_{0}\right) \in 2 \pi \mathbb{Z} \text { for all } m_{0} \in M \cap W\right\}
$$

and this set is a discrete union of translates of $W^{\perp}$.

It is enough to prove (2) when $C$ is simplicial. Indeed, we can always subdivide $C$ by simplicial cones $C_{j}$, and then $\Theta(C)$ is a sum (with signs) of the generalized functions $\Theta\left(C_{j}\right)$. Now consider a simplicial cone $C \subset V$, the intersection of $n$ distinct hyperplanes $\left(w_{k}, x\right) \geq 0$ with $w_{k}$ in the lattice $N$ of $V^{*}$. Let $w^{k} \in V$ be the 
dual basis of $w_{k}$. An element $x \in V$ is written as $x=\sum \eta_{k} w^{k}$, with $\eta_{k}=\left(w_{k}, x\right)$. Thus

$$
C=\bigoplus_{k} \mathbb{R}^{+} w^{k}
$$

Let $L=\bigoplus_{k=1}^{n} \mathbb{Z} w^{k}$ be the lattice of $V$ spanned by $w^{k}$. We have

$$
L \cap C=\bigoplus_{k} \mathbb{Z}^{+} w^{k}
$$

Let $\chi$ be a multiplicative character of $L$. Consider the discrete measure

$$
h(C, \chi, L)=\sum_{m \in L \cap C} \chi(m) \delta(m)
$$

and its Fourier transform

$$
\Theta(C, \chi, L)(y)=\sum_{m \in L \cap C} \chi(m) e^{i(m, y)} .
$$

Formula (3.6) gives

$$
\prod_{k=1}^{n}\left(1-\chi\left(w^{k}\right) e^{i\left(w^{k}, y\right)}\right) \Theta(C, \chi, L)(y)=1 .
$$

This equation implies that $\Theta(C, \chi, L)$ is smooth outside the zeroes of the analytic function $g(y)=\prod_{k=1}^{n}\left(1-\chi\left(w^{k}\right) e^{i\left(w^{k}, y\right)}\right)$. This zero set is a union of a discrete set of hyperplanes. Thus we obtain

Lemma 3.5. For y outside a union of a discrete set of hyperplanes,

$$
\Theta(C, \chi, L)(y)=\frac{1}{\prod_{k=1}^{n}\left(1-\chi\left(w^{k}\right) e^{i\left(w^{k}, y\right)}\right)} .
$$

With the notation as above, a basis of the dual lattice $L^{*}$ to $L$ consists of $w_{1}, \ldots, w_{n}$. Let $T=V^{*} / L^{*}=V^{*} /\left(\bigoplus_{k} \mathbb{Z} w_{k}\right)$. Then $T$ is an $n$-dimensional torus. Characters of $L$ are parametrized by $T$ : an element $g \in T$ gives a character $\chi_{g}$ by writing $\chi_{g}(x)=e^{2 i \pi(x, y)}$ if $x \in L$ and if $y \in V^{*}$ represents $g \in V^{*} / L^{*}$.

Consider the finite subgroup $G=N /\left(\bigoplus_{k} \mathbb{Z} w_{k}\right) \subset T$. Recall that $N$ is the dual lattice to $M$. Thus, for $x \in L$,

$$
\begin{array}{ll}
\sum_{g \in G} \chi_{g}(x)=0, & \text { if } x \notin M, \\
\sum_{g \in G} \chi_{g}(x)=|G|, & \text { if } x \in M .
\end{array}
$$

We obtain

$$
\delta(M \cap C)=|G|^{-1} \sum_{g \in G} h\left(C, \chi_{g}, L\right) .
$$

From Lemma 3.5, we obtain

Lemma 3.6. Let $C \subset V$ be a rational simplicial cone. Then, for y outside a union of a discrete set of hyperplanes, we have

$$
\Theta(C)(y)=|G|^{-1} \sum_{g \in G} \frac{1}{\prod_{k=1}^{n}\left(1-\chi_{g}\left(w^{k}\right) e^{i\left(w^{k}, y\right)}\right)} .
$$


This explicit formula for simplicial cones implies Proposition 3.4. To check that $\phi(y)$ is a rational function of $e^{y}$, let us state another formula for $\Theta(C)(y)$. Let $\alpha^{1}, \ldots, \alpha^{n}$ be the primitive vectors of $M$ on the edges $\mathbb{R}^{+} w^{1}, \ldots, \mathbb{R}^{+} w^{n}$ of $C$. Then the set

$$
S(C)=M \cap\left\{\sum_{k=1}^{n} t_{k} \alpha^{k} \mid 0 \leq t_{k}<1\right\}
$$

is finite and

$$
\Theta(C)(y) \cdot \prod_{k=1}^{n}\left(1-e^{i\left(\alpha^{k}, y\right)}\right)=\sum_{m \in S(C)} e^{i(m, y)}
$$

In the sequel, we will say that a property holds for generic $y \in V^{*}$ if there exists some nonzero analytic function $g$ such that the property holds for all $y$ with $g(y) \neq 0$.

Let $P$ be a lattice polytope. Consider the Fourier transform of identities (1), (2), and (3) of Proposition 3.2. By definition, the Fourier transform of $\delta(M \cap P)$ is the function $y \mapsto D(P)(i y)$. We choose an integral element $v_{0} \in M$ on each face $f$ of $P$. Then $M \cap C_{P}^{+}(f)=v_{0}+\left(M \cap C_{f}\right)$, while $M \cap C_{P}^{-}(f)=v_{0}-\left(M \cap C_{f}\right)$. We obtain the following proposition.

Proposition 3.7. We have the equality of generalized functions:

(1)

$$
D(P)(i y)=\sum_{f \in \mathcal{F}}(-1)^{\operatorname{dim} f} e^{i\left(v_{0}, y\right)} \Theta\left(C_{f}\right)(y)
$$

$$
(-1)^{n} D\left(P^{0}\right)(i y)=\sum_{f \in \mathcal{F}}(-1)^{\operatorname{dim} f} e^{i\left(v_{0}, y\right)} \Theta\left(C_{f}\right)(-y),
$$

$$
1=\sum_{f \in \mathcal{F}}(-1)^{\operatorname{dim} f} \Theta\left(C_{f}\right)(y)
$$

For each vertex $s$, consider the acute cone $C_{s}$. Proposition 3.4 shows that there exists a meromorphic function $\phi\left(C_{s}\right)$ on $V_{\mathbb{C}}^{*}$ (in fact, a rational function of $e^{y}$ ) such that $\Theta\left(C_{s}\right)(y)=\phi\left(C_{s}\right)(i y)$ for $y$ generic.

Proposition 3.8. We have the equalities of meromorphic functions on $V_{\mathbb{C}}^{*}$ :

(1)

$$
D(P)(y)=\sum_{s \in \mathcal{F}(0)} e^{(s, y)} \phi\left(C_{s}\right)(y)
$$

$$
D\left(P^{0}\right)(y)=(-1)^{n} \sum_{s \in \mathcal{F}(0)} e^{(s, y)} \phi\left(C_{s}\right)(-y)
$$


Furthermore

$$
\sum_{s \in \mathcal{F}(0)} \phi\left(C_{s}\right)(y)=1
$$

Proof. Consider formulae (1) and (2) of Proposition 3.7. When $f \in \mathcal{F}$ is a face of strictly positive dimension, the cone $C_{f}$ contains the nonzero linear vector space $\langle f\rangle$, and $\Theta\left(C_{f}\right)$ is supported on a union of affine spaces of dimension strictly less than $n$. Thus we obtain the identities above for $y \in i V^{*}$ generic. The last identity is obtained from relation (3).

Consider a simple lattice polytope $P$ with associated fan $\Sigma$. In this case, we have explicit expressions for the meromorphic functions $\phi\left(C_{s}\right)$. Indeed $C_{s}$ is the intersection of the $n$ half-spaces $\left(u_{F}, y\right) \geq 0, F \in \mathcal{F}^{s}$. The elements $u_{F}$ belong to $N$. Let $\sigma_{s} \in \Sigma$ be the polar cone to $C_{s}$. Recall the definitions of $U(\sigma)$ and of $G(\sigma)$ by formulae (2.3) and (2.4). The lattice $U\left(\sigma_{s}\right)$ is the lattice with $\mathbb{Z}$-basis $\left(u_{F}, F \in \mathcal{F}^{s}\right)$ and the group $G\left(\sigma_{s}\right)$ is the group $N / U\left(\sigma_{s}\right)$. Let $\left(m_{s}^{F}, F \in \mathcal{F}^{s}\right)$ be the dual basis to $\left(u_{F}, F \in \mathcal{F}^{s}\right)$. Applying Lemma 3.6, we obtain

$$
\phi\left(C_{s}\right)(y)=\frac{1}{\left|G\left(\sigma_{s}\right)\right|} \sum_{g \in G\left(\sigma_{s}\right)} \frac{1}{\prod_{F \in \mathcal{F}^{s}}\left(1-\chi_{g}\left(m_{s}^{F}\right) e^{\left(m_{s}^{F}, y\right)}\right)} .
$$

This leads to the following explicit formulae for $D(P)$ and $D\left(P^{0}\right)$ as a sum of meromorphic functions attached to each vertex $s$ of $P$. These formulae are the generalization of formula (3.2) in the 1-dimensional case.

Proposition 3.9. For $y \in V_{\mathbb{C}}^{*}$ generic, we have

$$
\sum_{m \in M \cap P} e^{(m, y)}=\sum_{s \in \mathcal{F}(0)} \frac{e^{(s, y)}}{\left|G\left(\sigma_{s}\right)\right|} \sum_{g \in G\left(\sigma_{s}\right)} \frac{1}{\prod_{F \in \mathcal{F}^{s}}\left(1-\chi_{g}\left(m_{s}^{F}\right) e^{\left(m_{s}^{F}, y\right)}\right)},
$$

while

$$
\sum_{m \in M \cap P^{0}} e^{(m, y)}==(-1)^{n} \sum_{s \in \mathcal{F}(0)} \frac{e^{(s, y)}}{\left|G\left(\sigma_{s}\right)\right|} \sum_{g \in G\left(\sigma_{s}\right)} \frac{1}{\prod_{F \in \mathcal{F}^{s}}\left(1-\chi_{g}\left(m_{s}^{F}\right) e^{-\left(m_{s}^{F}, y\right)}\right)} .
$$

Let $P$ be a simple polytope in $V$. We now analyze the continuous version

$$
E(P)(y)=\int_{P} e^{(x, y)} d x
$$

of $D(P)$. We do not assume that $P$ is a lattice polytope, as we will have to consider deformed polytopes $P(h)$. Let $s$ be a vertex of $P$. We choose inward pointing normal vectors $\left(u_{F}, F \in \mathcal{F}^{s}\right)$ and dual elements $\left(m_{s}^{F}, F \in \mathcal{F}^{s}\right)$. Then the volume of the parallelepiped constructed on $\left(m_{s}^{F}, F \in \mathcal{F}^{s}\right)$ is equal to $\left|\operatorname{det}\left(m_{s}^{F}\right)\right|_{F \in \mathcal{F}^{s} \text {. The }}$ following formula expresses the analytic function $E(P)$ as a sum over all vertices of meromorphic functions attached to each vertex $s$ of $P$. This is the $n$-dimensional analogue of formula (3.1).

Proposition 3.10. Let $P$ be a simple polytope. Let $y \in V_{\mathbb{C}}^{*}$ be such that $\left(m_{s}^{F}, y\right) \neq$ 0 for all vertices $s$ and all $F \in \mathcal{F}^{s}$. Then

$$
\int_{P} e^{(x, y)} d x=(-1)^{n} \sum_{s \in \mathcal{F}(0)} e^{(s, y)}\left(\left|\operatorname{det}\left(m_{s}^{F}\right)\right|_{F \in \mathcal{F}^{s}}\right) \frac{1}{\prod_{F \in \mathcal{F}^{s}}\left(m_{s}^{F}, y\right)}
$$


Proof. It is possible to give a direct argument for this proposition using Proposition 3.1 and explicit formulas for Fourier transforms of characteristic functions of simplicial cones. However, we can also deduce the value of $E(P)$ from the value of $D(P)$ by a limit argument using Riemann sums to evaluate an integral. Indeed, it is sufficient to prove this formula for lattice polytopes (choosing lattice $M$ with smaller and smaller fundamental domain). We have

$$
E(P)(y)=\lim _{q \rightarrow \infty} q^{-n} \sum_{m \in(M / q) \cap P} e^{(m, y)}
$$

when $q$ becomes a large integer.

We replace $M$ by $M / q$ in the formula of Proposition 3.9. We obtain

$$
q^{-n} \sum_{m \in(M / q) \cap P} e^{(m, y)}=\sum_{s \in \mathcal{F}(0)} \frac{e^{(s, y)}}{\left|G\left(\sigma_{s}\right)\right|} \sum_{g \in G\left(\sigma_{s}\right)} \frac{1}{\prod_{F \in \mathcal{F}^{s}} q\left(1-\chi_{g}\left(m_{s}^{F}\right) e^{\left(m_{s}^{F}, y / q\right)}\right)} .
$$

We see that only the trivial term $g=1$ in each group $G\left(\sigma_{s}\right)$ will contribute to the limit at $q=\infty$, and we obtain our proposition, as we observe that $\left|G\left(\sigma_{s}\right)\right|^{-1}$ is the absolute value of $\operatorname{det}\left(m_{s}^{F}\right)_{F \in \mathcal{F}^{s}}$.

In particular, we have $\operatorname{vol}(P)=\lim _{t \rightarrow 0} E(P)(t y)$, and we obtain that for any generic $y$,

$$
\operatorname{vol}(P)=\frac{(-1)^{n}}{n !} \sum_{s \in \mathcal{F}(0)}\left(\left|\operatorname{det}\left(m_{s}^{F}\right)\right|_{F \in \mathcal{F}^{s}}\right) \frac{(s, y)^{n}}{\prod_{F \in \mathcal{F}^{s}}\left(m_{s}^{F}, y\right)} .
$$

Let $P$ be a simple lattice polytope with facets $F_{1}, F_{2}, \ldots, F_{d}$. Let $h=\left(h_{1}, \ldots, h_{d}\right)$ be a small parameter of deformation.

Lemma 3.11. Let $\phi$ be a polynomial function on $V$. For $h \in \mathbb{R}^{d}$ small, the function $I(\phi)(h)=\int_{P(h)} \phi(x) d x$ is polynomial in $h$.

Proof. Consider

$$
E(y)(h)=E(P(h))(y)=\int_{P(h)} e^{(x, y)} d x .
$$

We compute $E(y)(h)$ using Proposition 3.10. Let $s$ be a vertex of $P$. Let $\sigma_{s}$ be the polar cone to $C_{s}$. We have

$$
\sigma_{s}=\sum_{j, F_{j} \in \mathcal{F}^{s}} \mathbb{R}^{+} u_{j}
$$

The subset of $\{1,2, \ldots, d\}$ consisting of those $j$ with $F_{j} \in \mathcal{F}^{s}$ is the set $\mathcal{E}\left(\sigma_{s}\right)$ of Definition 2.6. We denote by $\left(m_{s}^{j}, j \in \mathcal{E}\left(\sigma_{s}\right)\right)$ the dual basis to $\left(u_{j}, j \in \mathcal{E}\left(\sigma_{s}\right)\right)$. When $h$ is small, the point $s(h)$ given by

$$
s(h)=s-\sum_{j \in \mathcal{E}\left(\sigma_{s}\right)} h_{j} m_{s}^{j}
$$

is a vertex of $P(h)$. Thus, for generic $y$,

$$
E(y)(h)=\sum_{s \in \mathcal{F}(0)} E(s, y)(h),
$$


where

$$
E(s, y)(h)=\frac{(-1)^{n}}{\left|G\left(\sigma_{s}\right)\right|} \frac{e^{\left(s-\sum_{j \in \mathcal{E}\left(\sigma_{s}\right)} h_{j} m_{s}^{j}, y\right)}}{\prod_{j \in \mathcal{E}\left(\sigma_{s}\right)}\left(m_{s}^{j}, y\right)} .
$$

The function $E(y)(h)$ is analytic in $y$. Considering the Taylor expansion of $t \mapsto$ $E(t y)(h)$ at $t=0$, we obtain for every $k$ and generic $y$,

$$
\frac{1}{k !} \int_{P(h)}(x, y)^{k} d x=\frac{(-1)^{n}}{(n+k) !} \sum_{s \in \mathcal{F}(0)} \frac{\left((s, y)-\sum_{j \in \mathcal{E}\left(\sigma_{s}\right)} h_{j}\left(m_{s}^{j}, y\right)\right)^{n+k}}{\left|G\left(\sigma_{s}\right)\right| \prod_{j \in \mathcal{E}\left(\sigma_{s}\right)}\left(m_{s}^{j}, y\right)} .
$$

The polynomial behaviour in $h$ of $\int_{P(h)}(x, y)^{k} d x$ is apparent from this formula. As this result holds for any generic $y$ and any $k$, we obtain our lemma.

Remark that the Todd operator $\operatorname{Todd}(a, \partial / \partial h)$ is well defined on functions $h \mapsto$ $e^{h z}$ provided $z$ is sufficiently small. We rewrite also formula (3.9) as

$$
E(y)(h)=\sum_{s \in \mathcal{F}(0)} E(s, y)(h)
$$

with

$$
E(s, y)(h)=(-1)^{n}\left(\prod_{j \in \mathcal{E}\left(\sigma_{s}\right)} e^{-h_{j}\left(m_{s}^{j}, y\right)}\right) \frac{e^{(s, y)}}{\left|G\left(\sigma_{s}\right)\right|} \prod_{j \in \mathcal{E}\left(\sigma_{s}\right)} \frac{1}{\left(m_{s}^{j}, y\right)} .
$$

This shows that $\operatorname{Todd}(\Sigma, \partial / \partial h)$ is well defined on

$$
E(y)(h)=\int_{P(h)} e^{(x, y)} d x
$$

provided that $y$ is sufficiently small.

Theorem 3.12. Let $P$ be a simple lattice polytope, and let $\Sigma$ be the associated fan. If $y \in V_{\mathbb{C}}^{*}$ is small, then

$$
\left.\operatorname{Todd}(\Sigma, \partial / \partial h)\left(\int_{P(h)} e^{(x, y)} d x\right)\right|_{h=0}=\sum_{m \in M \cap P} e^{(m, y)},
$$

while

$$
\left.\operatorname{Todd}(\Sigma,-\partial / \partial h)\left(\int_{P(h)} e^{(x, y)} d x\right)\right|_{h=0}=\sum_{m \in M \cap P^{0}} e^{(m, y)} .
$$

Consider the Taylor expansion of both members of the first equality above at $y=0$. We obtain

$$
\left.\operatorname{Todd}(\Sigma, \partial / \partial h)\left(\int_{P(h)}(x, y)^{k} d x\right)\right|_{h=0}=\sum_{m \in M \cap P}(m, y)^{k}
$$

for all $y \in V_{\mathbb{C}}^{*}$ and $k \in \mathbb{N}$. Thus Theorem 3.12 implies Theorem 2.15.

Proof. Consider formula (3.11) for the function $E(y)(h)$. For $s$ a vertex of $P$, the function $E(s, y)(h)$ depends only of the variables $h_{j}$ such that $j \in \mathcal{E}\left(\sigma_{s}\right)$. Let $k$ be such that $k \notin \mathcal{E}\left(\sigma_{s}\right)$. From formula $(2.7)$, we see that $\operatorname{Todd}\left(a^{k}, \partial / \partial h_{k}\right) E(s, y)(h)=$ 0 if $a^{k} \neq 1$, while if $a^{k}=1$, we have $\operatorname{Todd}\left(1, \partial / \partial h_{k}\right) E(s, y)(h)=E(s, y)(h)$. By 
Lemma 2.13, if $\gamma \in \Gamma_{\Sigma}$ is not in $G\left(\sigma_{s}\right)$, then there is $k \notin \mathcal{E}\left(\sigma_{s}\right)$ such that $a^{k}(\gamma)$ is not 1. Thus $\operatorname{Todd}(\gamma, \partial / \partial h) E(s, y)(h)=0$ if $\gamma \notin G\left(\sigma_{s}\right)$. We obtain

$$
\operatorname{Todd}(\Sigma, \partial / \partial h) E(s, y)(h)=\sum_{\gamma \in G\left(\sigma_{s}\right)} \operatorname{Todd}(\gamma, \partial / \partial h) E(s, y)(h),
$$

and for $\gamma \in G\left(\sigma_{s}\right)$,

$$
\operatorname{Todd}(\gamma, \partial / \partial h) E(s, y)(h)=\left(\prod_{j \in \mathcal{E}\left(\sigma_{s}\right)} \operatorname{Todd}\left(a^{j}(\gamma), \partial / \partial h_{j}\right)\right) E(s, h) .
$$

We have

$$
\left.\operatorname{Todd}(a, \partial / \partial h) e^{u h}\right|_{h=0}=\operatorname{Todd}(a, u)=\frac{u}{1-a e^{-u}} .
$$

We obtain, for $\gamma \in G\left(\sigma_{s}\right)$,

$$
\begin{aligned}
& \left.\operatorname{Todd}(\gamma, \partial / \partial h) E(s, y)(h)\right|_{h=0} \\
& =(-1)^{n} \prod_{j \in \mathcal{E}\left(\sigma_{s}\right)} \frac{-\left(m_{s}^{j}, y\right)}{\left(1-a^{j}(\gamma) e^{\left(m_{s}^{j}, y\right)}\right)} \frac{e^{(s, y)}}{\left|G\left(\sigma_{s}\right)\right|} \prod_{j \in \mathcal{E}\left(\sigma_{s}\right)} \frac{1}{\left(m_{s}^{j}, y\right)} \\
& =\frac{e^{(s, y)}}{\left|G\left(\sigma_{s}\right)\right| \prod_{j \in \mathcal{E}\left(\sigma_{s}\right)}\left(1-a^{j}(\gamma) e^{\left(m_{s}^{j}, y\right)}\right)} .
\end{aligned}
$$

By the definition of $a^{j}, a^{j}(\gamma)=\chi_{\gamma}\left(m_{s}^{j}\right)$. Comparing with the first formula of Proposition 3.9, we obtain the first formula of our theorem. By a similar proof, we obtain the second formula.

\section{The COEFficients of the Ehrhart POLYNomial}

Let $P$ be a convex lattice polytope in $V$ with nonempty interior $P^{0}$. Consider, for $q$ a positive integer, the polytope $q P$. Let $\phi$ be a function on $V$. Let

$$
i(\phi, P)(q)=\sum_{m \in M \cap(q P)} \phi(m)
$$

and

$$
i\left(\phi, P^{0}\right)(q)=\sum_{m \in M \cap\left(q P^{0}\right)} \phi(m) .
$$

As a consequence of Proposition 3.8, let us prove the following generalization of a well-known theorem of Ehrhart.

Proposition 4.1. If $\phi$ is a homogeneous polynomial function of degree $k$, then the functions $q \mapsto i(\phi, P)(q)$ and $q \mapsto i\left(\phi, P^{0}\right)(q)$ are polynomial of degree $n+k$. Moreover, we have

$$
i\left(\phi, P^{0}\right)(q)=(-1)^{n+k} i(\phi, P)(-q)
$$

and

$$
i(\phi, P)(0)=\phi(0) .
$$


Proof. Observe that the vertices of $q P$ are the $q s$ ( $s$ a vertex of $P$ ) and that the tangent cone at $q s$ to $q P$ is $C_{s}$. Therefore, using Proposition 3.8, we obtain for generic $y$ :

$$
\sum_{m \in M \cap(q P)} e^{(m, y)}=\sum_{s \in \mathcal{F}(0)} e^{(q s, y)} \phi\left(C_{s}\right)(y)
$$

and

$$
\sum_{m \in M \cap\left(q P^{0}\right)} e^{(m, y)}=(-1)^{n} \sum_{s \in \mathcal{F}(0)} e^{(q s, y)} \phi\left(C_{s}\right)(-y) .
$$

Now replace $y$ by $t y$ for small nonzero $t$ and consider the expansion into Laurent series in $t$. As $\phi\left(C_{s}\right)(y)$ is of order at least $-n$, we have $\phi\left(C_{s}\right)(t y)=\sum_{j \geq-n} t^{j} a_{j}^{s}(y)$, where $a_{j}^{s}(y)$ are homogeneous rational functions of degree $j$. We thus see that

$$
\frac{1}{k !} \sum_{m \in M \cap(q P)}(m, y)^{k}=\sum_{s \in \mathcal{F}(0)} \sum_{j=0}^{k+n} \frac{1}{j !} q^{j}(s, y)^{j} a_{k-j}^{s}(y)
$$

and that

$$
\frac{1}{k !} \sum_{m \in M \cap\left(q P^{0}\right)}(m, y)^{k}=(-1)^{n} \sum_{s \in \mathcal{F}(0)} \sum_{j=0}^{k+n} \frac{1}{j !} q^{j}(s, y)^{j}(-1)^{k-j} a_{k-j}^{s}(y) .
$$

We thus obtain the polynomial behaviour in $q$ of $i(\phi, P)(q)$ and of $i\left(\phi, P^{0}\right)(q)$ for the polynomial function $\phi(x)=(x, y)^{k}$ and the first identity as well. As this result holds for all $y$ and $k$, it holds for all polynomial functions on $V$. We also obtain, for $q=0$ and for $\phi(x)=(x, y)^{k}$, that

$$
\sum \frac{t^{k}}{k !} i(\phi, P)(0)
$$

is equal to the Laurent series expansion of $\sum_{s \in \mathcal{F}(0)} \phi\left(C_{s}\right)(t y)$. Thus we obtain the second identity from formula (3) of Proposition 3.8 as we have identically $\sum_{s \in \mathcal{F}(0)} \phi\left(C_{s}\right)=1$.

For $\phi=1$, the polynomial $i(\phi, P)(q)$ is called the Ehrhart polynomial. We denote it simply by $i(P)$. We write

$$
i(P)(q)=|M \cap(q P)|=\sum_{k=0}^{n} q^{k} a_{k}(P) .
$$

It follows from Proposition 4.1 that the term $a_{0}(P)$ is equal to 1 .

Let us give, for example, the values of $a_{k}(P)$ for the simplex $P(a, b, c)$ considered in Example 2.9. Let $p$ and $q \geq 1$ be two coprime integers. Let $s(p, q)$ be the Dedekind sum, defined by

$$
s(p, q)=\sum_{i=1}^{q}\left(\left(\frac{i}{q}\right)\right)\left(\left(\frac{p i}{q}\right)\right),
$$

where $((x))=0$ if $x$ is integral and $((x))=x-[x]-\frac{1}{2}$ otherwise.

We have

$$
a_{3}(P)=a b c / 6, \quad a_{2}(P)=(a b+b c+c a+1) / 4, \quad a_{0}(P)=1,
$$


while $a_{1}(P)$ is equal to

$$
\frac{1}{12}\left(\frac{a b}{c}+\frac{b c}{a}+\frac{c a}{b}+\frac{1}{a b c}\right)+(a+b+c) / 4-s(b c, a)-s(c a, b)-s(a b, c)+3 / 4
$$

This formula, originally due to Mordell, has been generalized recently by Pommersheim [9] and Kantor-Khovanskii [6]: more generally, they computed the coefficient $a_{n-2}(P)$ of the Ehrhart polynomial. Let us show how to deduce their results from Theorem 2.15, which gives (in principle) an explicit formula for the Ehrhart polynomial of any simple polytope.

Let $P$ be a simple lattice polytope, and let $\Sigma$ be its fan. Consider the Todd operator $\operatorname{Todd}(\Sigma, \partial / \partial h)$. We write it as the sum of its homogeneous components

$$
\operatorname{Todd}(\Sigma, \partial / \partial h)=\sum_{k=0}^{\infty} A_{k}(\partial / \partial h)
$$

Lemma 4.2. We have

$$
a_{n-k}(P)=\left.A_{k}(\partial / \partial h) \operatorname{vol}(P(h))\right|_{h=0} .
$$

Proof. Let $q$ be a positive integer. We have

$$
|M \cap(q P)|=\left.T(\Sigma, \partial / \partial h) \operatorname{vol}((q P)(h))\right|_{h=0} .
$$

Formula (3.8) shows that for any generic $y$,

$$
\operatorname{vol}((q P)(h))=\frac{(-1)^{n}}{n !} \sum_{s \in \mathcal{F}(0)} \frac{\left((q s, y)-\sum_{j \in \mathcal{E}\left(\sigma_{s}\right)} h_{j}\left(m_{s}^{j}, y\right)\right)^{n}}{\left|G\left(\sigma_{s}\right)\right| \prod_{j \in \mathcal{E}\left(\sigma_{s}\right)}\left(m_{s}^{j}, y\right)}
$$

Thus the lemma follows.

Let us write

$$
\operatorname{Todd}(\Sigma, \partial / \partial h)=\operatorname{Todd}(\partial / \partial h)+R(\partial / \partial h)
$$

with

$$
\operatorname{Todd}(\partial / \partial h)=\prod_{j=1}^{d} \operatorname{Todd}\left(\partial / \partial h_{j}\right)
$$

and

$$
R(\partial / \partial h)=\sum_{\gamma \in \Gamma_{\Sigma}, \gamma \neq 1} \operatorname{Todd}(\gamma, \partial / \partial h)
$$

We write

$$
\operatorname{Todd}(\partial / \partial h)=\sum_{k=0}^{\infty} T_{k}(\partial / \partial h)
$$

and

$$
R(\partial / \partial h)=\sum_{k=0}^{\infty} R_{k}(\partial / \partial h),
$$

where $T_{k}, R_{k}$ are homogeneous polynomials of degree $k$.

We have thus

$$
a_{n-k}(P)=m_{n-k}(P)+r_{d-k}(P)
$$


with

$$
m_{n-k}(P)=\left.T_{k}(\partial / \partial h) \cdot \operatorname{vol}(\Delta(h))\right|_{h=0}
$$

and

$$
r_{n-k}(P)=\left.R_{k}(\partial / \partial h) \cdot \operatorname{vol}(\Delta(h))\right|_{h=0} .
$$

Lemma 4.3. We have

$$
T_{0}(\partial / \partial h)=I, \quad T_{1}(\partial / \partial h)=\frac{1}{2} \sum_{j=1}^{d} \partial / \partial h_{j},
$$

while

$$
R_{0}(\partial / \partial h)=0, \quad R_{1}(\partial / \partial h)=0 .
$$

Proof. The first two equalities follow readily from formula (2.6).

The groups $G(\sigma)$ are trivial for $\sigma \in \Sigma(1)$. Thus there is no element $\gamma$ of $\Gamma_{\Sigma}$ with $a_{k}(\gamma)=1$ for all $k$ but one, and the last equalities follow from formula (2.7).

More generally, by the same argument, we obtain the following

Lemma 4.4. Assume $G(\sigma)=\{1\}$ for all cones $\sigma \in \Sigma$ of dimension at most $K$. Then $R_{k}(\partial / \partial h)=0$ and hence $a_{n-k}(P)=m_{n-k}(P)$ for all $k \leq K$.

Let $f \in \mathcal{F}$ be a face of $P$. Consider the vector space $\langle f\rangle$ and its lattice $M \cap\langle f\rangle$. We denote by $\operatorname{vol}(f)$ the volume of the face $f$ with respect to the Lebesgue measure on $\langle f\rangle$ determined by this lattice.

Let $f$ be a face of codimension 2 . Then $f$ is the intersection of two facets. To simplify notation, we assume that $f=F_{1} \cap F_{2}$. Then $\sigma_{f}$ (the polar cone of $C_{f}$ ) is generated by two normal vectors $u_{1}$ and $u_{2}$ to $F_{1}$ and $F_{2}$. The elements $u_{1}, u_{2}$ generate a sublattice $U\left(\sigma_{f}\right)$ of $N \cap\left\langle\sigma_{f}\right\rangle$. We have

$$
G\left(\sigma_{f}\right)=\left(N \cap\left\langle\sigma_{f}\right\rangle\right) / U\left(\sigma_{f}\right) .
$$

As $u_{1}$ is primitive, we can always choose a $\mathbb{Z}$-basis $n_{1}, n_{2}$ of $N \cap\left\langle\sigma_{f}\right\rangle$ such that $u_{1}=n_{1}$ and $u_{2}=p n_{1}+q n_{2}$ with $1 \leq p \leq q$. The integers $(p, q)$ are coprime. We have $q=|G(\sigma)|$. Recall formula (4.1) for $s(p, q)$.

Definition 4.5. Let $f$ be a face of codimension 2. Using notation above, define

$$
\mu(f)=\frac{1}{4}-\frac{1}{4 q}+s(p, q) .
$$

Proposition 4.6 ([9], [6]). We have

$$
\begin{gathered}
m_{n}(P)=\operatorname{vol}(P), \quad r_{n}(P)=0, \\
m_{n-1}(P)=\frac{1}{2} \sum_{F \in \mathcal{F}(n-1)} \operatorname{vol} F, \quad r_{n-1}(P)=0 \\
r_{n-2}(P)=\sum_{f \in \mathcal{F}(n-2)} \mu(f) \operatorname{vol}(f) .
\end{gathered}
$$


Proof. Let $\sigma$ be an element of the fan $\Sigma$. Define

$$
e(\sigma, \partial / \partial h)=\prod_{j \in \mathcal{E}(\sigma)} \partial / \partial h_{j}
$$

From formula (4.2), we readily obtain

Lemma 4.7. Let $f$ be a face of $P$, and $\sigma_{f} \in \Sigma$ the corresponding cone. Then we have

$$
\left.e\left(\sigma_{f}, \partial / \partial h\right) \operatorname{vol}(P(h))\right|_{h=0}=\left|G\left(\sigma_{f}\right)\right|^{-1} \operatorname{vol}(f) .
$$

The values of $a_{n}(P), a_{n-1}(P)$ are well known and easily obtained from Lemmas 4.3 and 4.7. It remains to obtain the value of $r_{n-2}(P)$. Consider the subset $\Gamma_{2}$ of $\Gamma_{\Sigma}$ defined by

$$
\Gamma_{2}=\bigcup_{\sigma \in \Sigma(2)} G(\sigma)
$$

Let $\Gamma_{2}^{\prime}=\Gamma_{2}-\{1\}$. We have

$$
R_{2}(\partial / \partial h)=\sum_{\gamma \in \Gamma_{2}^{\prime}} \operatorname{Todd}(\gamma, \partial / \partial h) .
$$

For $\sigma \in \Sigma(2)$, let $G(\sigma)^{\prime}=G(\sigma)-\{1\}$. Then $\Gamma_{2}^{\prime}$ is the disjoint union of the sets $G(\sigma)^{\prime}$ when $\sigma$ varies in $\Sigma(2)$. We study

$$
R_{2}(\sigma, \partial / \partial h)=\sum_{\gamma \in G(\sigma)^{\prime}} \operatorname{Todd}(\gamma, \partial / \partial h)
$$

for $\sigma \in \Sigma(2)$. To simplify notation, we write $\sigma=\mathbb{R}^{+} u_{1}+\mathbb{R}^{+} u_{2}$, and as before we choose a $\mathbb{Z}$-basis $n_{1}, n_{2}$ of $\langle\sigma\rangle \cap N$ such that $u_{1}=n_{1}, u_{2}=p n_{1}+q n_{2}$. Elements of $G(\sigma)=(\langle\sigma\rangle \cap N) / U(\sigma)$ are represented by elements $j n_{2}$ with $0 \leq j<q$. If $\gamma=j n_{2}$, we write $\gamma=-(j p / q) u_{1}+(j / q) u_{2}$. By definition $a^{k}(\gamma)=1$ except for $k=1,2$. We have $a^{1}(\gamma)=e^{-2 \pi i j p / q}$, while $a^{2}(\gamma)=e^{2 i \pi j / q}$. Thus by formula (2.7)

$$
R_{2}(\sigma, \partial / \partial h)=\left(\sum_{j=1}^{q-1}\left(1-e^{-2 \pi i j p / q}\right)^{-1}\left(1-e^{2 i \pi j / q}\right)^{-1}\right)\left(\partial / \partial h_{1}\right)\left(\partial / \partial h_{2}\right) .
$$

If $f$ is the face of $P$ such that $\sigma=\sigma_{f}$, we have by Lemma 4.7,

$$
\left.\left(\partial / \partial h_{1}\right)\left(\partial / \partial h_{2}\right) \operatorname{vol}(P(h))\right|_{h=0}=q^{-1} \operatorname{vol}(f) .
$$

By formula (18a) of [10], we have

$$
\begin{aligned}
& q^{-1} \sum_{j=1}^{q}\left(1-e^{2 i \pi j p / q}\right)^{-1}\left(1-e^{-2 i \pi j / q}\right)^{-1} \\
& \quad=-s(-p, q)+\frac{q-1}{4 q}=s(p, q)-\frac{1}{4 q}+\frac{1}{4} .
\end{aligned}
$$

Thus we obtain

$$
R_{2}\left(\sigma_{f}\right) \operatorname{vol}(P(h))_{h=0}=\mu(f) \operatorname{vol}(f) .
$$

Summing over all faces of codimension 2 , we obtain the desired formula for $r_{n-2}(P)$. 


\section{REFERENCES}

[1] M. F. Atiyah, Elliptic operators and compact groups, Lecture Notes in Mathematics, vol. 401, Springer, 1974. MR 58:2910

[2] S. E. Cappell, J. L. Shaneson, Genera of algebraic varieties and counting lattice points, Bull. A.M.S. (New series) 30 1994, 62-69. MR 94f:14018

[3] W. Fulton, Introduction to toric varieties, Ann. of Math. Study 131, Princeton University Press, 1993. MR 94g:14028

[4] V. Ginzburg, V. Guillemin and Y. Karshon, Cobordism techniques in symplectic geometry, The Carus Mathematical Monographs, Mathematical Association of America, to appear.

[5] M. N. Ishida, Polyhedral Laurent series and Brion's equalities, International Journal of Math. 1 1990, 251-265. MR 91m:14081

[6] J. M. Kantor, A. G. Khovanskii, Une application du théorème de Riemann-Roch combinatoire au polynôme d'Ehrhart des polytopes entiers de $\mathbb{R}^{d}$, C. R. Acad. Sci. Paris, Série I 317 1993, 501-507. MR 94k:52018

[7] T. Kawasaki, The Riemann-Roch theorem for complex V-manifolds, Osaka J. Math. 16 1979, 151-159. MR 80f: 58042

[8] A. G. Khovanskii, A. V. Pukhlikov, A Riemann-Roch theorem for integrals and sums of quasipolynomials over virtual polytopes, St. Petersburg Math. J. 4 1993, 789-812. MR 94c: 14044

[9] J. Pommersheim, Toric varieties, lattice points and Dedekind sums, Math. Ann. 2951993 , 1-24. MR 94c: 14043

[10] H. Rademacher, E. Grosswald, Dedekind sums, The Carus Mathematical Monographs, 16, Mathematical Association of America, 1972. MR 50:9767

[11] G. Ziegler, Lectures on polytopes, Graduate Texts in Mathematics, vol. 152, Springer-Verlag, 1995. MR 96a:52011

Ecole Normale Supérieure de Lyon, 46 allée d'Italie, 69364 Lyon Cedex 07, France

E-mail address: mbrion@fourier.ujf-grenoble.fr

DMi, Ecole Normale Supérieure, 45 rue d'Ulm, 75005 Paris, France

E-mail address: vergne@dmi.ens.fr 